\title{
MBSE-Entwicklungsfähigkeit für Digitale Zwillinge
}

\author{
Thomas C. Zimmermann, \\ Christian Masuhr und \\ Rainer Stark, Berlin
}

Das Konzept des Digital Twins nutzt Modelle über die Entwicklung und Absicherung hinaus auch für Betriebsoptimierungen. Modellbasiertes Systems Engineering (MBSE) ermöglicht eine durchgängige Systemmodellierung mit offenen Schnittstellen für die Systementwicklung. Dieser Beitrag stellt an einem beispielhaften IoT-Pumpensystem Schnittstellenerweiterungen vor, um MBSE für eine durchgängige Modellierung, von der Entwicklung über den Betrieb bis Endof-Life, nutzen zu können. Die gewonnenen Informationen können zurückgeführt und für zukünftige Produktneuentwicklungen genutzt werden. hergestellt werden [3]. Das Resultat sind digitale Repräsentationen von realen Produkten oder Produkt-Service-Systemen - inklusive ihres dynamischen Verhaltens.

Beim dokumentenbasierten Systems Engineering liegen die Architekturspezifikationen als statisches funktionales Modell vor, was dazu führt, dass die $\mathrm{Zu}-$ rückverfolgbarkeit von Problemen eingeschränkt ist und ein großes Risiko von Fehleinträgen besteht, was zu mangelhafter Konsistenz der Informationen führen kann. Mit modellbasierten Systems Engineering wird ein umfassendes Systemmodell, oft auch Single Source of Truth genannt, aufgebaut, aus dem die jeweils benötigten Sichtweisen auf das System dynamisch abgeleitet werden können. Das Konzept des „Digital Thread" verbindet die Aspekte des klassischen MBSE (Modellbasiertes Systems Engineering)durchgängig mit der Systemintegration und Absicherung, bedeutet aber auch zunehmende Anforderungen an IT-Infrastrukturen. Schnittstellen und modulare Modellierungsarchitekturen können für die Anbindung an den digitalen Schatten genutzt werden. Die Informationsfabrik stellt Infrastruktur für den benötigten Austausch von Daten zwischen Digital Master und Shadow bereit und beschreibt die Gesamtheit der notwendigen Informationssystemschichten (z.B. PLM, ERP, SCM, IoT) und Kompetenzen in der Betreiberumgebung der Zukunft [4].

\section{Die Entwicklung und Nutzung von Digital Twins}

Im Rahmen der Digital Twin Entwicklung stellen die Entwicklungselemente Digital Master und Digital Twin, sowie die Verlinkung zwischen beiden Aspekten die größten Einflussfaktoren zur erfolgreichen Realisierung des Digital Twins dar [5]. Darüber hinaus wirken diese Elemente besonders auf die Digital Twin Aspekte „Model Richness“ und „Simulation Capabilities" und weisen also einen starken Modellierungs- und somit Systembezug mit hoher Komplexität auf weshalb anders als bei anderen Aspekten des Digital Twins grundsätzlich systemspezifische Lösungen erforderlich sind. Um Mehrwerte der Digital-Twin-Technologien abzuschöpfen, ist die spezifische Umsetzung aller Digital-Twin-Elemente für das betrachtete System erforderlich. Dies führt tementwicklung durch die notwendige jeweils Use Case bezogene Ausmodellierung und Simulation des Systems.

Der Digital Master stellt ein umfassendes Systemmodell bereit, das instanziiert und mit den Daten aus dem Digital Shadow äußere Einflüsse aus realen Systemdaten erfährt, da nur so gewährleistet werden kann, dass der Digital Twin eine hinreichende Gesamtmenge an Betriebszuständen, Umwelteinflüssen und Verhaltensweisen des Systems adäquat abbilden kann. Zur Darstellung von Mechatronischen Systemen ist deshalb die Abbildung zu Mehraufwänden im Nachgang der Sys- 
von Geometrien sowie logischem und physikalischem Systemverhalten in dynamischen und statischen Modellen erforderlich. Außerdem ist eine Ad-hoc-Simulation notwendig, um einen hinreichenden Digital Twin bereitzustellen. Darüber hinaus sind präskriptive Methoden gefragt, um durch simulierte Szenarien Vorhersagen über Systemkonfigurationen, Betriebszustände und Umwelteinflüsse treffen $\mathrm{zu}$ können. Häufig ist die Interpretation von Sensordaten und Anreicherung mit dem entsprechenden Kontext entscheidend, da die erzeugten Daten alleine zunächst keinen Mehrwert bieten. Erst ihre Verknüpfung mit Produktdaten, z. B. über Systemmodelle, führen insbesondere bei komplexen Systemen zu einem wesentlichen Mehrwert und Wettbewerbsvorteil für die Anwender. Die im Rahmen von MBSE genutzten Artefakte, wie z.B. Modelle und Simula-tionen, können als Grundlage für die Erstellung des Digital Twins genutzt werden [6]. Darüber hinaus lässt sich der Digital Shadow durch die Nutzung von MBSE effizienter aufbauen, da die durchgängige Modellierung Teile der Datenkontextualisierung übernimmt und im Zusammenspiel mit der Informationsfabrik bereits Schnittstellen zur Verlinkung on Shadow und Master bietet.

\section{| Digital Twins mit MBSE}

Um den Aufwand der Digital Twin Entwicklung zu reduzieren, ist es notwendig, die verschiedenen Elemente bereits in der Systementwicklung und -integration mitzudenken. Modellbasiertes Systems Engineering als Systementwicklungskonzept erscheint geeignet zu sein, besonders den Digital Master, aber auch den Digital Shadow sowie die Verlinkung dieser beiden Elemente maßgeblich zu unterstützen. Im Rahmen von MBSE können die beiden DTDimensionen „Digital Model Richness“ und „Simulation Capabilities“ von Synergien profitieren, indem das Konzept der Datendurchgängigkeit mit MBSE über die Systementwicklung hinaus in den gesamten nachgelagerten Lebenszyklus getragen wird, um Digital Twins effizienter zu entwickeln und zu betreiben. MBSE-Entwicklungsfähigkeiten lassen sich in fünf grundlegende Aspekte gliedern (Tabelle 1):

- Systems Environment Analytics,

- System Definition und Derivation,

- Systems Interaction Modeling,

- Systems Lifecycle Engineering sowie

MBSE Capability and Maturation Matrix.

Tabelle 1. MBSE-Dimensionen mit Entwicklungsfähigkeiten und Digital Twin Synergien

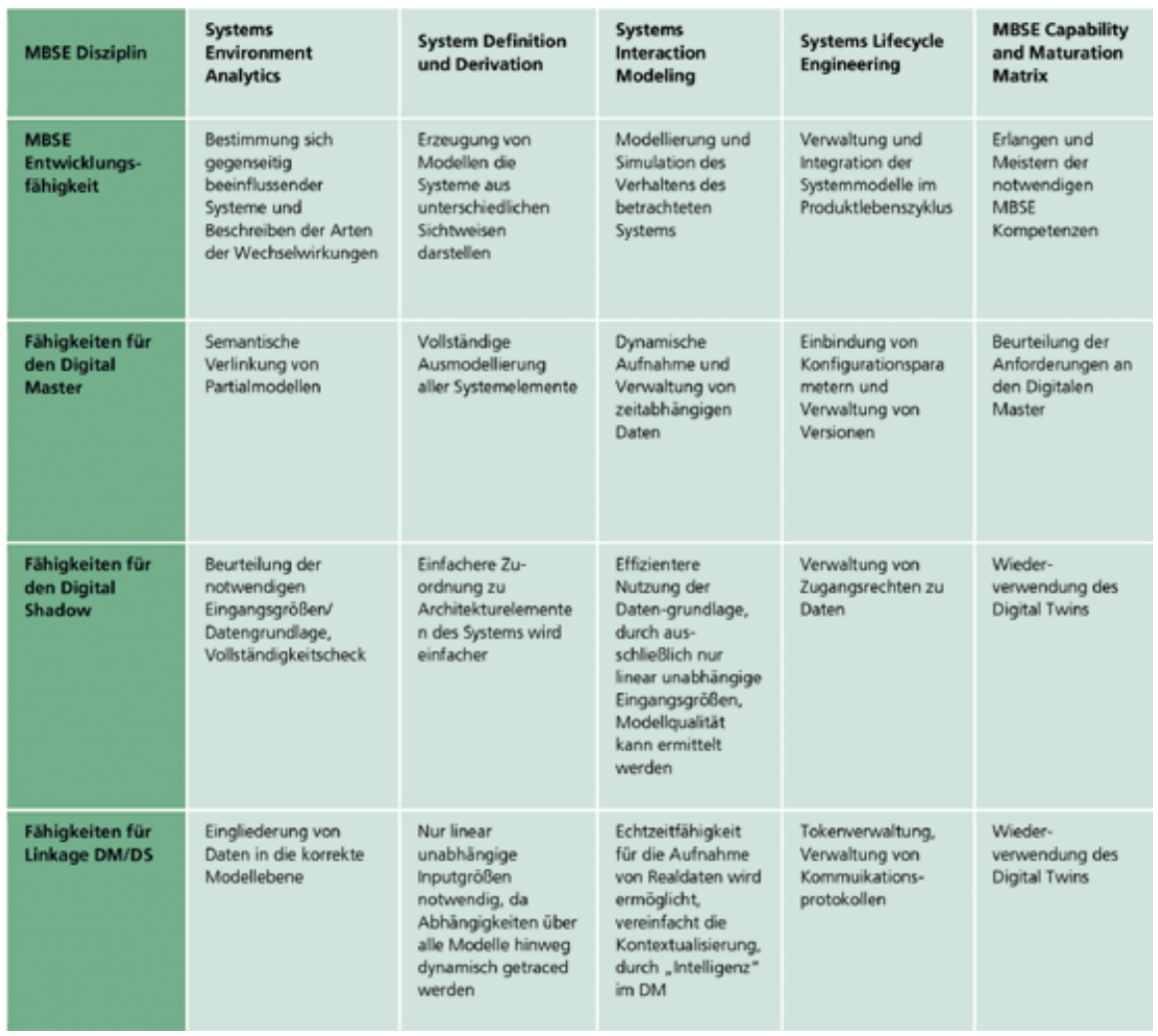

Diese fünf MBSE-Disziplinen liefern prozess- und methodenunabhängige Entwicklungsfähigkeiten, die für das modellbasierte Entwickeln komplexer Systeme benötigt und für die effizientere Entwicklung von Digital Twins herangezogen werden können. Zur Identifikation der tatsächlichen Potenziale von MBSE für die Entwicklung von Digital Twins müssen diese in Bezug zu den relevanten Entwicklungselementen des Digital Twins gesetzt werden. In Tabelle 1 wird ersichtlich, wo Synergien bezüglich der DT Elemente bestehen. Hierbei wird deutlich, dass die digitale Durchgängigkeit und die umfassende Modellierung eines Systems in mehreren Partialmodellen, die mit MBSE ermöglicht werden, für DT, DS und die Verlinkung beider Elemente signifikante Fähigkeiten bereitstellen. Der starke Fokus auf die Identifikation von externen und internen Systemschnittstellen und die Etablierung einer durchgängigen Modelllandschaft und Datendurchgängigkeit durch Tracing-Konzepte, die auch Ad-hoc-Simulationen eines oder mehrerer verknüpfter Modelle ermöglichen, sowie die starke Modularisierung, die simulierte Systemintegration ohne DomainExpertenwissen ermöglicht, unterstützen die Fähigkeit. Modelle nicht nur mit anderen Modellen, sondern im Rahmen des
Digital Twins auch mit Betriebsdaten aus dem Digital Shadow zu betreiben.

Die Nutzung dynamisch verknüpfter bietet darüber hinaus die Möglichkeit die Datenmenge im Digital Shadow auf tatsächlich unabhängige Variablen zu reduzieren, da sich gegenseitig beeinflussende Faktoren über die Modellarchitektur aus der MBSE-Entwicklung über die gesamte Modellarchitektur propagiert werden. Dadurch wird auch die Zahl der erforderlichen Schnittstellen zwischen Digitalem Schatten und Digitalem Master insgesamt reduziert, da Daten ausschließlich referenziert werden und nie doppelt vorliegen. Die Entwicklungsfähigkeiten von MBSE ermöglichen es darüber hinaus, mit Digital Twins nicht nur Fehlerszenarien und neue Systemkonfigurationen virtuell $\mathrm{zu}$ analysieren, sondern die Rückkoppelung von Betriebsdaten des physischen Twins ermöglicht auch die Anpassung und Verbesserung der im MBSE genutzten Systemmodelle [7]. Dies kann zum Beispiel durch den Vergleich zwischen simuliertem Verhalten und Realdaten aus dem Betrieb realisiert werden, welche zum Beispiel durch IoT-Devices aufgenommen und im Rahmen der Informationsfabrik über die vorhandenen MBSE-Modellschnittstellen bereitgestellt werden. 


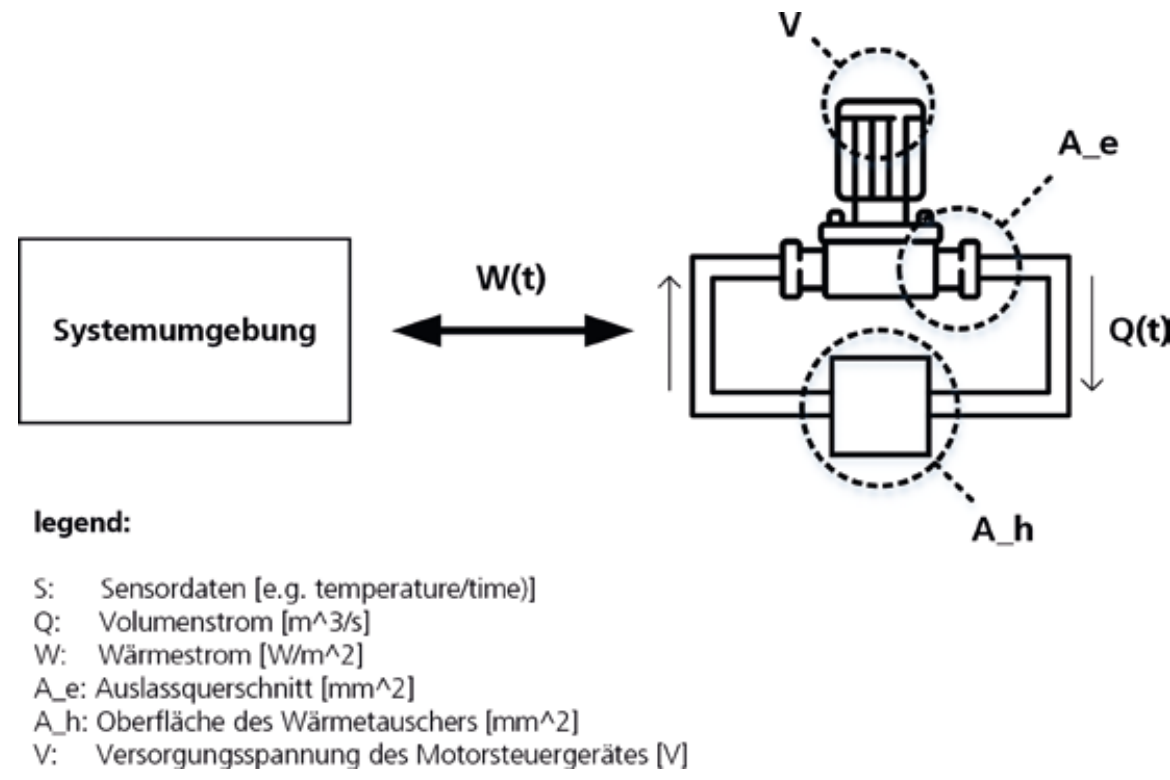

Bild 1. Pumpensystem und Systemumgebung

\section{Der Digitale Zwilling am Bei- spiel eines loT-Pumpensystems}

Am Beispiel einer Pumpe können sinnvolle Messgrößen einer integrierten IoTLösung anhand des Verwendungszwecks der Daten ableitet werden. Daten könnten bei einer End-of-Line-Kontrolle in der Montage oder während der Betriebsphase bei dem Kunden erzeugt werden und Informationen über die Fertigungsqualität oder den Systemzustand der Pumpe liefern. Ein stark vereinfachter Pumpenkreislauf besteht aus einer Pumpe samt Kreislauf, in dem ein Wärmeübertragungsmedium durch ein System befördert wird (Bild 1). Das verknüpfte System kann zum Beispiel die Beheizung eines Raumes darstellen oder einen Produktionsprozess mit Wärmeenergie versorgen. Der gesamte Pumpenkreislauf tauscht kontinuierlich Wärme mit seiner Umgebung aus. Da die zur Verfügung stehende Wärmeenergie im Kreislauf und die Wärmeabgabe an die Umgebung sich mit der Zeit verändern, wird der Pumpenkreislauf geregelt.

Partialmodelle aus der Systementwicklung mit MBSE sind:

- Systemumgebungsmodell,

- Anforderungsmodell,

- Funktionsmodell,

- Strukturmodell,

- Verhaltensmodell sowie

physikalische Modelle.

Messdaten für den Digitalen Schatten sind:
- Drücke,

- Motordrehzahl,

Volumenstrom,

- Temperaturen,

- Einsatzzeitpunkt und Betriebsstundenzähler,

- Strom sowie

- Spannung.

Anforderungs-, Funktions-, Strukturund Verhaltensmodelle werden über frühe Phasen des Produktentwicklungsprozesses hinaus verwendet und mit physikalischen Modellen aus späteren Entwicklungsschritten als Digitaler Master zusammengeführt.

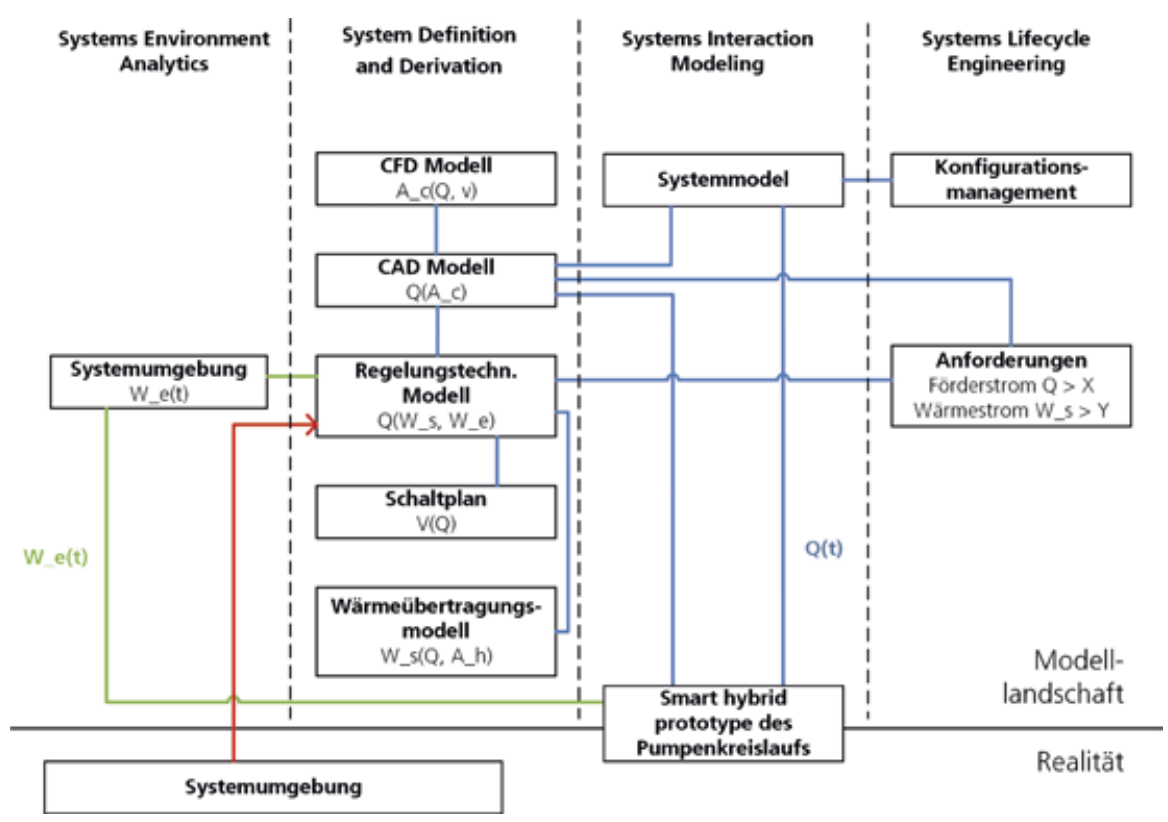

Bild 2. Modelllandschaft für den Digital Twin der Pumpe
Der Förderstrom Q (Volumen pro Zeit) ist ein mögliches Auslegungskriterium einer Pumpe und somit für den Digital Twin des Systems im späteren Betrieb von Interesse. Zusätzlich sind der Wärmestrom aus dem modellierten Umgebungsmodell und die Implementierung von beliebigen Sensordaten abgebildet. Im Rahmen der MBSE-Entwicklungsfähigkeiten können Real-Daten zum Beispiel durch ein IoT-Device übermittelt worden sein und spiegeln Zustände der realen Umgebung mithilfe der existierenden Entwicklungsmodelllandschaft wieder (Bild 2). Aufgrund der vereinfachten Darstellung wurde eine Vielzahl von Größen und Zusammenhängen an dieser Stelle vernachlässigt. Der konstruktive Aufbau einer an einer Pumpe wurde bei der Entwicklung mit MBSE in CAD-Daten gespeichert, welche durch ihre statischen Eigenschaften zwar - isoliert betrachtet - ein geringes Level der Digital Twin Dimension „Simulation Capabilites" erreichen, aber den Aspekt der „Digital Model Richness“ im Zusammenhang mit weiteren verknüpften Modellen deutlich unterstützen. Dennoch können Optimierungsergebnisse aus dynamischen Modellen notwendige Anpassungen des statischen Modells ermöglichen, indem durch eine geeignete Verknüpfung und die Rückführung von geometriespezifischen Größen ermöglicht wird. Anhand dieses einfachen Beispiels ist das Potenzial für die Entwicklungsfähigkeit der Verknüpfungen mit logischen und Verhaltensmodellen (z.B. regelungs- 
technische Modelle) deutlich, um Synergien zu erzeugen - besonders in komplexen Systemen. Messdaten, wie z.B. hydrostatischer Druck oder Volumenstrom, können dann in regelungstechnischen Modellen, die sich aus den verschiedenen physikalischen Partialmodellen zusammensetzen, als Digitaler Schatten verarbeitet werden, um in einer Instanz das Systemverhalten mittels Digitalem Zwilling möglichst realitätsgetreu abzubilden. Zusammen mit der Aufnahme der Temperatur ist die Verifikation von strömungsmechanischen, thermodynamischen Modellen und das Abbilden von Mechanismen der Wärmeübertragung möglich. Das Ableiten von Verschleißzuständen, welche beispielsweise das Resultat einer fehlerhaften Montage oder Auslegungsfehler im Design sind, können für erforderliche Designänderungen oder eine Echtzeitplanung von Wartungen verwendet werden. Die zugehörigen Modelle bilden durch den prädiktiven Charakter und der hohen Komplexität ein neues Level der Realisierung in den Dimensionen „Simulation Capabilites“ und „Digital Model Richness" eines Digital Twin. Auf diesem Umsetzungsraum kann mittels Smart Hybrid Prototyping neue Formen der Interaktion mit Nutzern ermöglicht werden. Darüber hinaus können Nutzungsinformationen (Einsatzzeitpunkt und Betriebsstundenzähler) im Rahmen des Digitalen Zwillings mit dem Stromverbrauch für ein Energiemanagement eingesetzt werden. Bei der Entsorgung ist eine Bewertung einzelne Komponenten oder Materialien aufgrund ihres Rohstoffgehalts oder Leistungsfähigkeit (z.B. elektrischeLeitfähigkeit) für eine Wiederverwendung denkbar.

\section{Schlussfolgerung und Ausblick}

Die Suche eines allgemeingültigen Digital-Twin Entwicklungsprozesses auf $\mathrm{Ba}^{-}$ sis von MBSE Methoden, welcher in der Lage ist die Verknüpfung aller möglichen Modelle und Anwendungsfälle gleichermaßen aus einem generischen Template für die zu betrachtende Systeminstanz abzuleiten, ist aufgrund der Einzigartigkeit sowohl des physischen Systems als auch der Systemmodellierung nicht zielführend. Trotz der bestehenden Limitierungen bietet MBSE für den digitalen Zwilling die Chance die digitale Durchgängigkeit auf die gesamte Lebensdauer des Systems sinnvoll zu erweitern. Dabei kann durch den zum Beispiel aus IoT-Sensordaten erzeugten digitalen Schatten nicht nur der aktuelle Systemzustand selber überwacht, sondern auch in Kombination mit dem Digitalen Master auf Basis von MBSE-Modellen anhand Digital Twin Methoden virtuell Betriebsszenarien evaluiert werden. Dadurch können nicht nur Vorhersagen über eine Systeminstanz getroffen werden, sondern auch die vorher erstellten Entwicklungsmodelle evaluiert werden. Auf diese Weise können sowohl das Systemkonzept als auch die Entwicklungsmodelle in folgenden Systemgenerationen verbessert werden. Durch die Nutzung von MBSE kann die die Erstellung digitaler Zwillinge vereinfacht und in Zukunft die digitale Durchgängigkeit nicht nur über die Systementwicklung, sondern über mehrere Produktgenerationen etabliert werden, um so einfacher aus Entwicklungsmodellen und -modellschnittstellen bessere digitale Zwillinge abzuleiten

\section{Literatur}

1. Shafto, M. et al.: Draft Modeling, Simulation, Information Technology \& Processing Roadmap. National Aeronautics and Space Administration, Technology Area 11, Washington, DC 2010

2. Stark, R.; Damerau, T.: Digital Twin. In: Chatti. S.; Tolio, T. (Hrsg.): CIRP Encyclopedia of Production Engineering. Springer-Verlag, Berlin, Heidelberg 2019, S. 1 - 8 DOI: 10.1007/978-3-642-35950-7_16870-1

3. Stark, R.; Kind, S.; Neumeyer, S.: Innovations in Digital Modelling for Next Generation Manufacturing System Design. CIRP Annals 66 (2017) 1, S. 169-172. DOI: 10.1016/j.cirp.2017.04.045

4. Damerau, T.: Informationsfabrik Industrie 4.0. Smart Data für die lebenszyklusbegleitende Informationslogistik. FUTUR (2016) 1. Online unter http://www.plmportal.org/de/ forschung-detail/informationsfabrik-industrie-4-0.html

5. Stark, R.; Fresemann, C.; Lindow, K.: Development and Operation of Digital Twins for Technical Systems and Services. CIRP Annals 68 (2019) 1, S. 129-132 DOI: 10.1016/j.cirp.2019.04.024

6. Dahmen, U.; Rossmann, J.: Experimentable Digital Twins for a Modeling and Simulation-based Engineering Approach. In: Proceedings of the $4^{\text {th }}$ IEEE International Symposium on Systems Engineering: October 1-3, 2018, Rome Marriott Park Hotel, Roma, Italy 2018, S. 1 - 8 DOI: 10.1109/SysEng.2018.8544383

7. Madni, A.; Madni, C.; Lucero, S.: Leveraging Digital Twin Technology in Model-Based Systems Engineering. Systems 7 (2019) 1, S. 7 DOI: $10.3390 /$ systems 7010007

\section{Die Autoren dieses Beitrags}

Thomas C. Zimmermann, M. Sc., M.Eng., geb.1985 studierte Luft- \& Raumfahrttechnik an der TU Berlin und Systems Engineering am Stevens Institute of Tech. in Hoboken, NJ, USA und war danach mehrere Jahre in der Luft- \& Raumfahrtindustrie im Bereich Systementwicklung tätig. Seit 2018 ist er als Wissenschaftlicher Mitarbeiter des Geschäftsfeldes Virtuelle Produktentstehung am Fraunhofer-Institut für Produktionsanlagen und Konstruktionstechnik IPK Berlin in der Abteilung Modellbasiertes Entwickeln tätig. Seine Forschungsschwerpunkte sind Modellbasiertes Systems Engineering, ganzheitliche Systemmodellierung, Funktionsarchitekturenund Systemabsicherung.

Christian Masuhr, M. Sc., geb. 1992 studierte Maschinenbau an der Universität Paderborn. Er arbeitet als Wissenschaftlicher Mitarbeiter des Geschäftsfeldes Virtuelle Produktentstehung am Fraunhofer-Institut für Produktionsanlagen und Konstruktionstechnik IPK Berlin in der Abteilung Modellbasiertes Entwickeln und ist dort seit 2019 tätig. Seine Forschungsschwerpunkte umfassen digitale Produktzwillinge, die digitale Fabrik und smarte hybride Prototypen für virtuelle Inbetriebnahmen, sowie das Verbessern von Produkten mittels Feedback in die Produktentwicklung.

Prof. Dr.-Ing. Rainer Stark, geb. 1964, studierte Maschinenbau an der Ruhr-Universität Bochum sowie der Texas A \& M University (USA). Von 1989 bis 1994 war er als Wissenschaftlicher Mitarbeiter am Lehrstuhl für Konstruktionstechnik/CAD der Technischen Fakultät der Universität des Saarlandes beschäftigt. Mit der Erlangung des Grades Dr.-Ing. wechselte er zur Ford AG. Dort war er zuletzt als Technischer Manager der „Virtuellen Produktentstehung und Methoden“ der Ford Motor Company Europa tätig. Seit Februar 2008 ist er Leiter des Fachgebietes Industrielle Informationstechnik der TU Berlin und Direktor des Geschäftsfeldes Virtuelle Produktentstehung des Fraunhofer-Instituts für Produktionsanlagen und Konstruktionstechnik.

\section{Summary}

MBSE Development Capabilities for Digital Twins. The Digital Twins concept uses models not only for development and protection, but also for operational optimization. MBSE enables integrated system modeling with open interfaces for system development. The work presents interface extensions on an exemplary IoT pump system in order to be able to use MBSE for end-to-end modelling, from development through operation to end-of-life. The information gained can be fed back and used for future product development.

\section{Bibliography}

DOI 10.3139/104.112312

ZWF 115 (2020) Special; page 51 -54

(C) Carl Hanser Verlag GmbH \& Co. KG ISSN 0947-0085 